\title{
Evaluación de la generación de sedimentos en el distrito de drenaje del Valle de
} Sibundoy, departamento del Putumayo (Colombia)*

Evaluation of Sediment Generation in the Drainage District of Valle de Sibundoy, Putumayo Province (Colombia)

Mario David Delgado-Gómez ${ }^{\text {a }}$

Universidad de Nariño, Colombia

DOI: https://doi.org/10.11144/Javeriana.ayd23-44.egsd

profemariodavid@gmail.com

ORCID: http://orcid.org/0000-0003-2394-5739

Fecha de recepción: 10 Septiembre 2018 Fecha de aprobación: 15 Noviembre 2018 Fecha de publicación: 30 Junio 2019

\section{Resumen:}

Esta investigación se desarrolló en el distrito de drenaje del Valle de Sibundoy, localizado a $1^{\circ} 12 \# 12 \# \mathrm{~N}$ y $76^{\circ} 51$ \# 15 \# O. Se realizó una simulación empleando el modelo hidrológico SWAT para estimar el balance hídrico y una calibración del modelo para caudales y sedimentos. Con la modelación hidrológica, se estimó un caudal promedio mensual para la cuenca de 32,26 m3/s. Para la producción de sedimentos, con las condiciones actuales de uso de suelo para la cuenca, se calculó un aporte de 1,18 T/ha/a. Con la ayuda de escenarios de cambio climático, se determinó que para el año 2046 se sobrepasará en más del 200\% la producción media de sedimentos. Finalmente, se proponen alternativas de mitigación de impactos, basadas en conservación de suelos, sistemas productivos sustentables, restauración de coberturas y gestión del riesgo en el recurso hídrico.

Palabras clave: drenaje, modelo, sedimentos, Sibundoy, SWAT.

\section{Abstract:}

This research was conducted in the drainage district of Valle de Sibundoy, located in $1^{\circ} 12 \# 12$ N N and $76^{\circ} 51$ \# $15 \#$ O. A simulation was carried out using the hydrological model SWAT to estimate water balance and the model was gauged for the water volumes and sediments. The hydrological modelling enabled to estimate the average monthly water volume for this basin at $32.26 \mathrm{~m} 3 / \mathrm{s}$. regarding the sediment production, under the current conditions of soil use for this basin, the water catchment was calculated at $1.18 \mathrm{~T} / \mathrm{ha} / \mathrm{a}$. Aided with climate change scenarios, it was determined that in 2046 the average sediment production will be exceeded in more than $200 \%$. Finally, some alternatives for mitigating the impacts are proposed, based on the soil preservation, sustainable productive systems, coverage restoration and risk management in the water resource.

Keywords: drainage, model, sediments, Sibundoy, SWAT model.

\section{Introducción}

Teniendo en cuenta que el distrito de drenaje es un sistema complejo, se observó la necesidad de implementar una herramienta para la evaluación del suelo y del agua en esta cuenca hidrográfica. Para esto, se recurrió al modelo Soil and Water Assessment Tool (SWAT) para predecir el impacto de las prácticas de manejo de suelo, la producción de agua y la generación de sedimentos, teniendo en cuenta variables como tipos de suelo, pendientes, coberturas y el sistema de drenaje de esta cuenca (Uribe, 2010).

Bajo la anterior premisa, y a través del modelo SWAT, se estimaron los caudales y los sedimentos en el sistema hidráulico de los canales que conforman el distrito de drenaje; se simularon unos escenarios de cambio climático que permitieran comparar la incidencia de las variables de cobertura en el caudal y la generación de sedimentos, y se concluyó con una configuración de alternativas de manejo del distrito basadas en una

Notas de autor

$$
\text { a Autor de correspondencia. Correo electrónico: profemariodavid@gmail.com }
$$


evaluación de impacto ambiental hecha con una matriz de Vicente Conesa (Secretaría Distrital de Integración Social, 2013).

Según Cortez (2004), el Valle de Sibundoy es susceptible a procesos erosivos, principalmente en aquellas zonas de pendientes moderadas a fuertes, con altos conflictos de uso, donde las zonas de ladera coinciden con un fallamiento transversal de dirección NO-SE que aumenta su susceptibilidad; así como también, por la alta presión de los suelos para explotaciones agropecuarias, cuya vocación es de protección absoluta. Por otra parte, la ampliación de la frontera agropecuaria en zonas de humedales o en áreas de influencia de estos ecosistemas estratégicos coincide con zonas no aptas para cultivar, por ser fácilmente inundables y con altos niveles freáticos. De acuerdo con lo anterior, esta investigación pretende determinar cuál es el valor promedio de la generación de sedimentos, expresados en T/ha/a, para el distrito de drenaje del Valle de Sibundoy.

Dentro de los elementos necesarios para implementar esta simulación, se pude mencionar a M. Silva (citado por Londoño y Zapata, 2013), quien afirma que los sedimentos, después de ser producidos, son transportados por una corriente para luego ser depositados. De acuerdo con su mecanismo de transporte, los sedimentos pueden estar en suspensión (los más finos, que llegan al cauce por el lavado de material suelto) o ser sólidos en estado de saltación, que se producen por causa de las irregularidades en el fondo.

Neitsch, Arnold, Kiniry y Williams (2005) mencionan que SWAT es un modelo que fue desarrollado por el doctor Jeff Arnold para el Departamento de Agricultura de los Estados Unidos (USDA, por sus siglas en inglés), para predecir el impacto de las prácticas de manejo del suelo en el agua, el sedimento y la producción de sustancias agrícolas químicas en cuencas grandes y complejas, con variedad de suelos, usos de la tierra y condiciones de manejo por un tiempo prolongado. Este modelo trabaja con unidades de respuesta hidrológica (HRU, por sus siglas en inglés), las cuales resultan del cruce de los tipos de suelo con las diferentes coberturas; a cada una de estas combinaciones se le atribuye un número para diferenciarlas y poder predecir el comportamiento del ciclo hidrológico, aplicando los principios de la ecuación universal de pérdida de suelos (cuya sigla en inglés es USLE), que predice el promedio de la tasa anual de erosión en una pendiente del terreno basada en el patrón de lluvia, el tipo de suelo, la topografía, los cultivos y las prácticas de manejo, teniendo en cuenta determinantes como la fuerza erosiva de la lluvia (factor $\mathrm{R}$ ), relacionada con la intensidad de la lluvia, el tipo de suelo (factor $\mathrm{K}$ ), el uso de la tierra y el manejo de cultivos (factor $\mathrm{C}$ ), las prácticas agrícolas (factor P) y las características topográficas (factor LS) (Srinivasan, 2013).

\section{Materiales y métodos}

\section{Localización}

Al suroccidente de Colombia, nororiente del departamento de Putumayo, en la subregión andinoamazónica, se encuentra ubicado el Valle de Sibundoy, localizado a $1^{\circ} 12^{\prime} 12^{\prime \prime} \mathrm{N}$ y $76^{\circ} 51^{\prime} 15^{\prime \prime} \mathrm{O}$ (figura 1), entre los $2000 \mathrm{~m} \mathrm{s.}$. m. m., en su parte plana, y los $3300 \mathrm{~m} \mathrm{s.} \mathrm{n.} \mathrm{m.,} \mathrm{en} \mathrm{la} \mathrm{parte} \mathrm{más} \mathrm{alta} \mathrm{(Corporación} \mathrm{para} \mathrm{el}$ Desarrollo del Sur de la Amazonia [Corpoamazonia] y Fundación Cultural del Putumayo, 2007).

\section{Modelo SWAT}

Este es un modelo basado en principios físicos que requiere de datos específicos acerca del clima, las propiedades físicas del suelo, la topografía, la vegetación y las prácticas de manejo del suelo. Por esta razón, fue necesario organizar los insumos para la simulación hidrológica teniendo en cuenta el flujograma propuesto por Ríos (2015), el cual se observa en la figura 2. 
División política del departamento del Putumayo

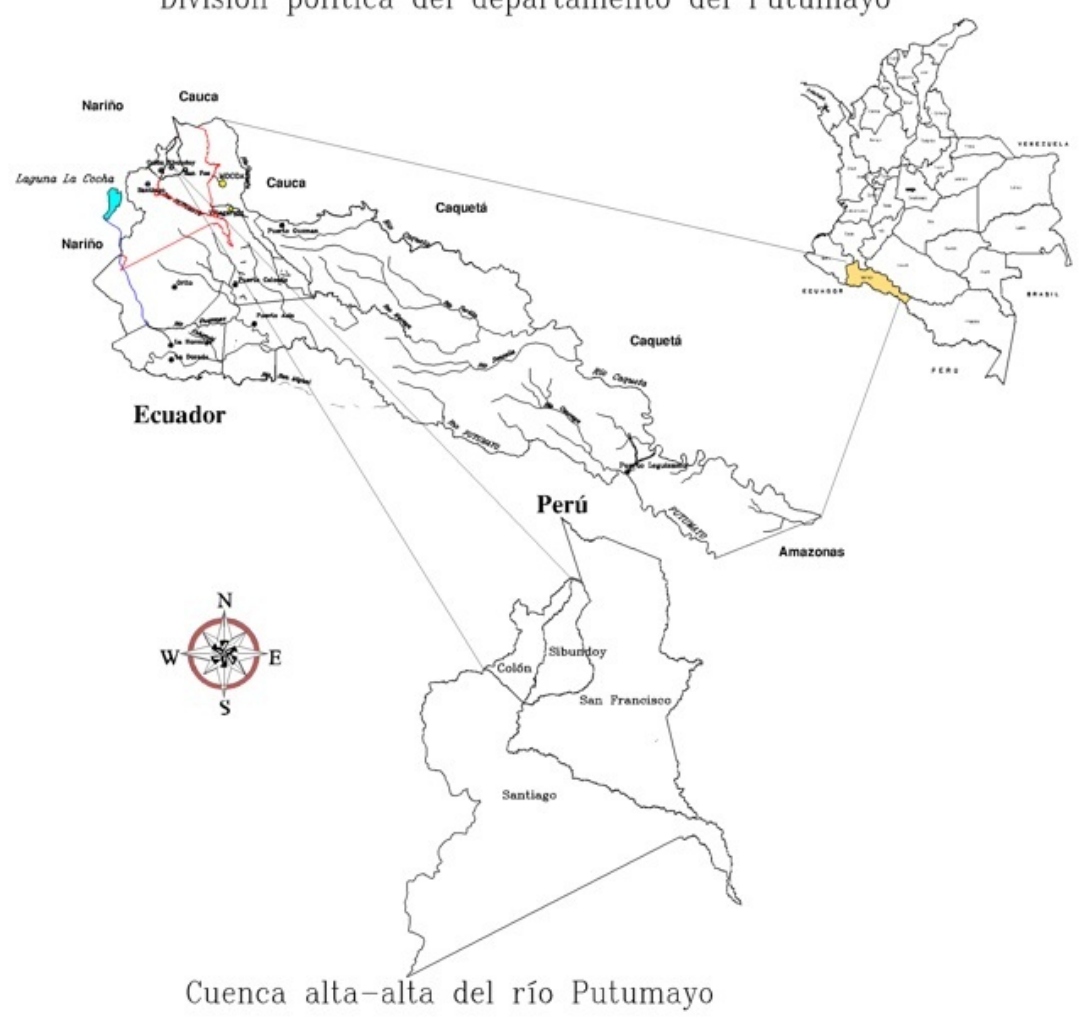

FIGURA 1.

Ubicación nacional, regional y local del distrito de drenaje del Valle de Sibundoy Fuente: Corpoamazonia (2008, p. 24)

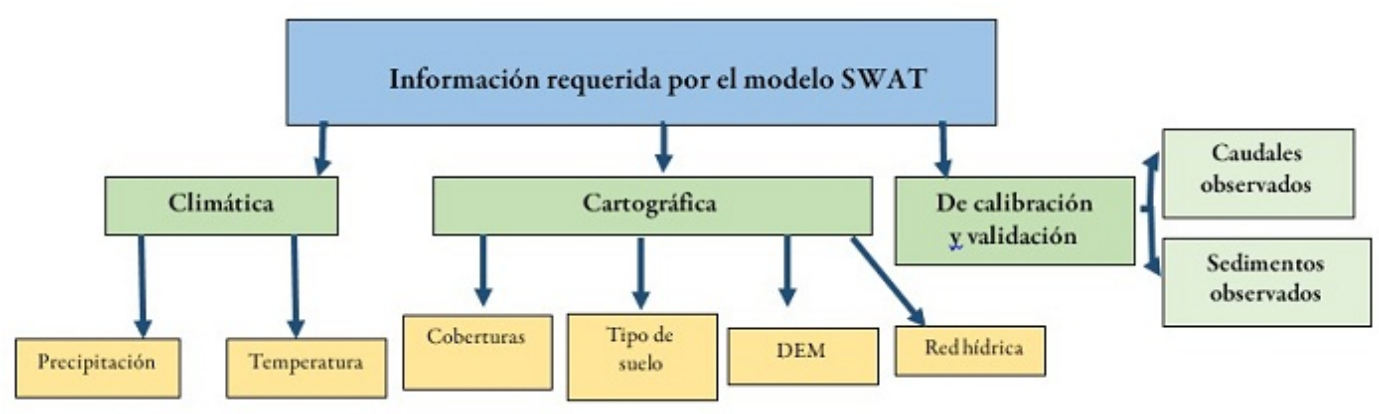

FIGURA 2.

Flujograma de los insumos requeridos por el modelo SWAT Fuente: Ríos (2015, p. 16)

\section{Análisis de escenarios de cambio climático sobre la producción de caudales y sedimentos}

Con el modelo SWAT se simularon dos condiciones de uso del suelo de las cuencas para predecir comportamientos futuros. El flujograma de la figura 3 resume la metodología empleada para obtener los caudales y los sedimentos para las condiciones actuales en un escenario positivo (B1) y en uno negativo (A2). Escamilla (2016) recomienda que la simulación y el modelo climático deben tener el mismo número de años proyectados. Para estimar las coberturas del año 2046, se utilizaron algunos indicadores relevantes, como tasa anual de deforestación, crecimiento urbano y ampliación de la frontera agrícola. 


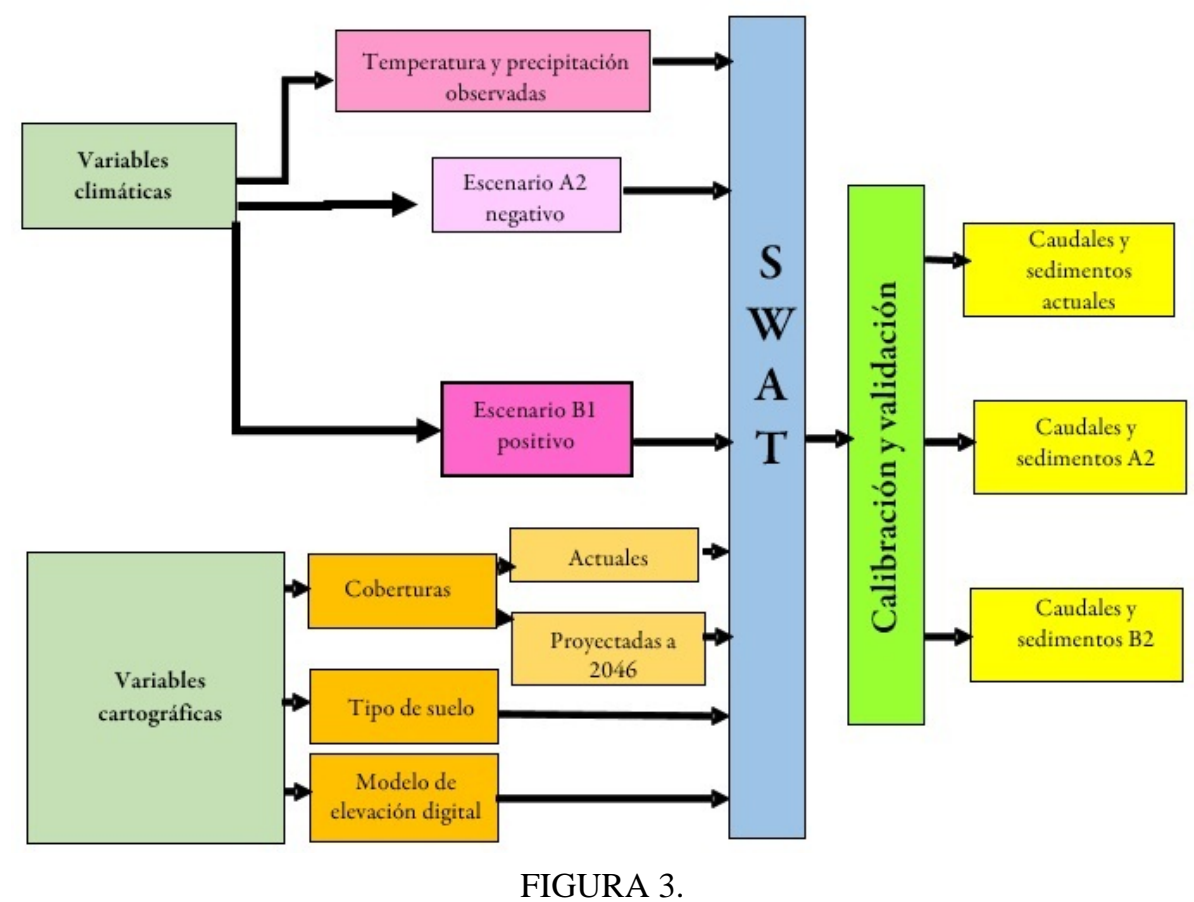

Flujograma para la obtención de caudales y sedimentos en los escenarios de cambio climático Fuente: elaboración propia

\section{Resultados}

\section{Balance hidrológico de la cuenca}

El modelo SWAT se basa en la ecuación del balance hídrico, la cual considera el agua que entra, la que se almacena y la que sale de la microcuenca (Solarte, 2014). Los valores promedio anuales obtenidos en esta investigación sobre el balance hídrico simulados por SWAT se presentan a continuación: 


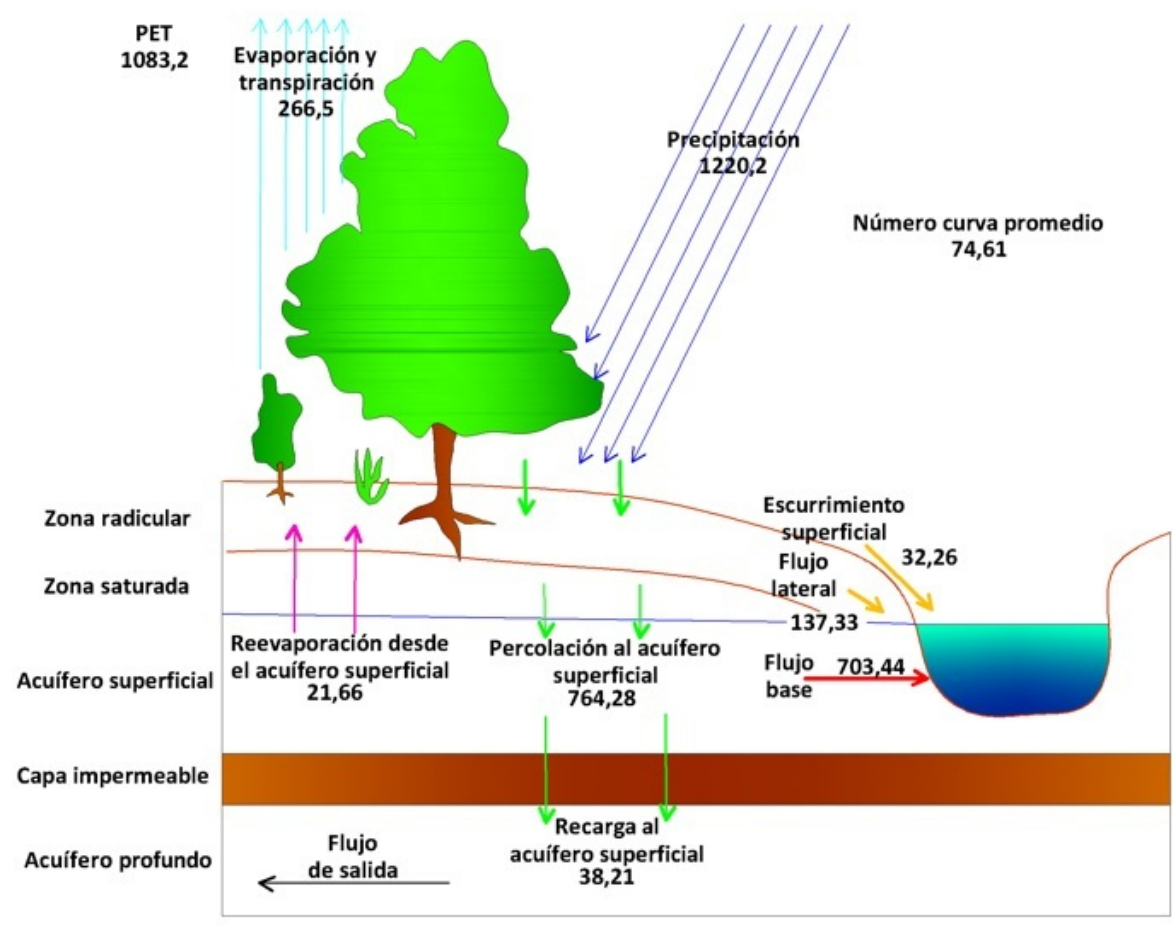

FIGURA 4.

Balance hidrológico para el distrito de drenaje del Valle de Sibundoy

Fuente: elaboración propia

Teniendo en cuenta la figura 4, y expresando el balance hidrológico en términos porcentuales, se encontró que, del total de la precipitación que cae sobre la cuenca, el 21,84\% vuelve a la atmósfera por evapotranspiración; la salida del sistema en forma de escorrentía superficial y subterránea representa el 75,03\%, mientras que el 3,13\% pasa a formar parte de la recarga de los acuíferos profundos.

\section{Calibración y Validación del modelo para caudal}

En la figura 5 se indica el comportamiento de los valores promedio mensuales del caudal. Con la validación del modelo se obtuvieron valores estadísticos favorables, que indican un coeficiente de determinación $\left(\mathrm{R}^{2}\right)$ de 0,83 y un coeficiente de eficiencia de Nash-Sutcliffe (NSE) de 0,76 , lo que cataloga a la simulación como muy buena, teniendo en cuenta la escala de valor propuesta por el modelo. 


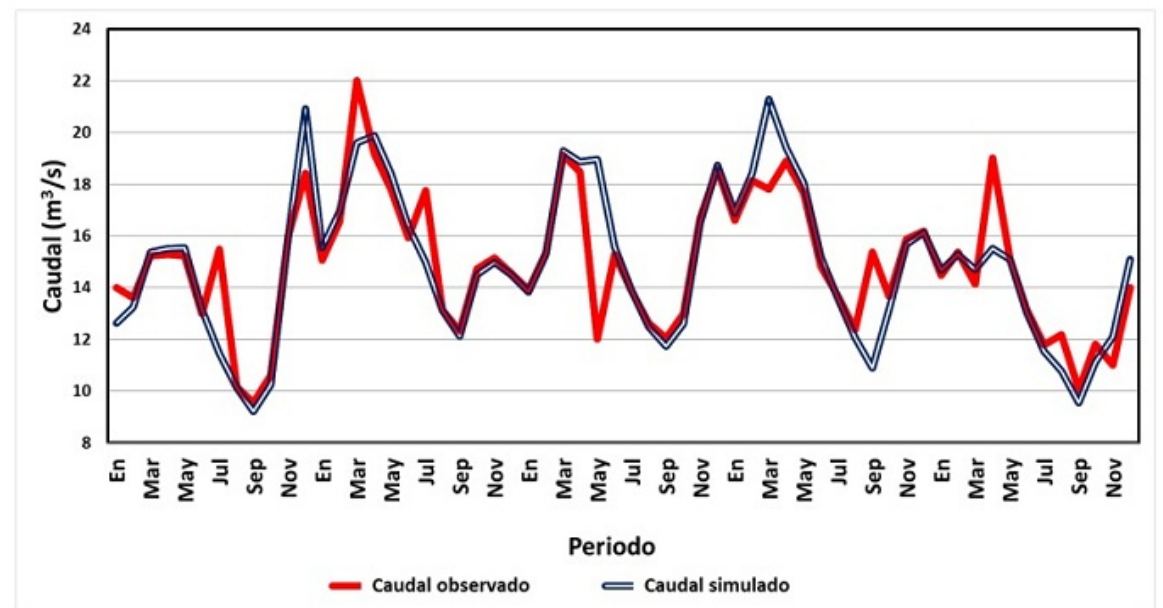

FIGURA 5.

Calibración y validación del modelo para caudal en el distrito de drenaje del Valle de Sibundoy Fuente: elaboración propia

La simulación hidrológica para el distrito de drenaje determinó que el caudal promedio de la cuenca en el punto de descarga, denominado la garganta del Balsayaco, fue de $32,26 \mathrm{~m}^{3} / \mathrm{s}$, donde el canal B aporta 16,89 $\mathrm{m}^{3} / \mathrm{s}$, que corresponde al $52,36 \%$, el canal A $11,96 \mathrm{~m}^{3} / \mathrm{s}$, que equivale al $37,07 \%$, y el cauce viejo del río Putumayo entrega $3,41 \mathrm{~m}^{3} / \mathrm{s}$, que corresponde al 10,57\%.

\section{Calibración y validación del modelo para sedimentos}

El modelo calcula la producción de sedimentos para cada HRU con la ecuación modificada de la pérdida universal de suelo (MUSLE, por sus siglas en inglés) (Londoño y Zapata, 2013).

Para los sedimentos, se utilizó el mismo intervalo de validación y calibración que para los caudales. En la figura 6, se indica el comportamiento de los valores promedio mensuales para la producción se sedimentos, expresados en $\mathrm{T} / \mathrm{ha} / \mathrm{a}$. Los valores estadísticos determinaron que la simulación fue buena, pues se obtuvo un $\mathrm{R}^{2}$ de 0,75 y un NSE de 0,68 . 


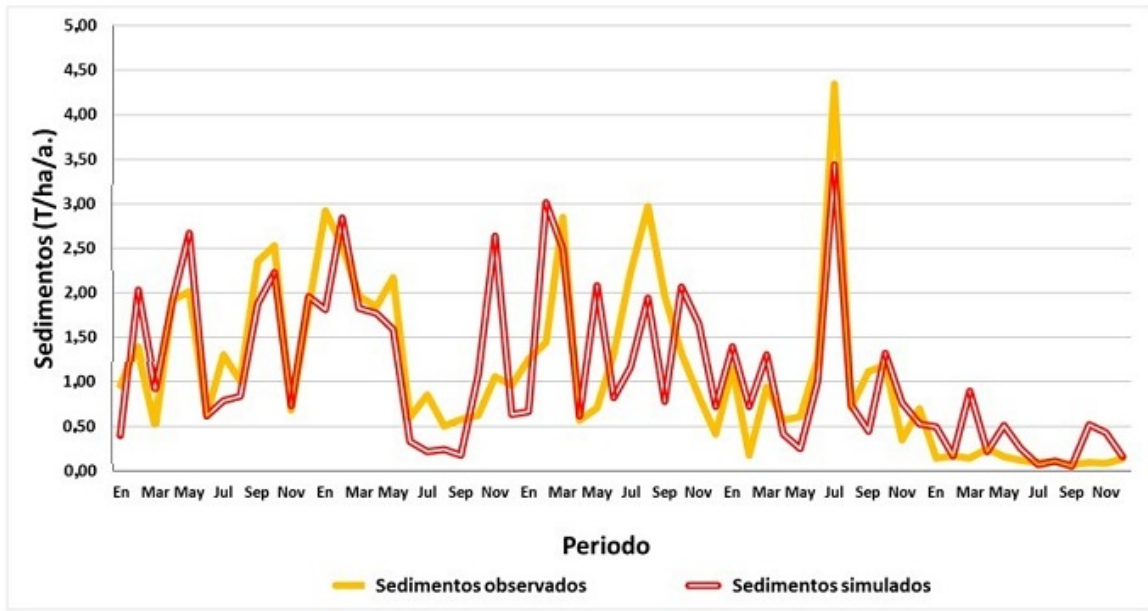

FIGURA 6.

Calibración y validación del modelo para producción de sedimentos en el distrito de drenaje del Valle de Sibundoy

Fuente: elaboración propia

La presente investigación determinó que en el punto de descarga de la cuenca del distrito de drenaje, denominado la garganta de Balsayaco, la producción promedio de sedimentos expresados fue de 1,18 T/ha/ a; donde el canal A aporta 0,14 T/ha/a, que corresponden al 12,20\%; el canal B, con 0,11 T/ha/a, aporta el 9,56\%, y el cauce viejo, con $0,06 \mathrm{~T} / \mathrm{ha} / \mathrm{a}$, aporta el 4,84\%.

\section{Simulación de caudales con base escenarios de cambio climático}

Los escenarios modelados propuestos arrojaron diferentes comportamientos en cuanto al escurrimiento superficial, existiendo diferencias estadísticas significativas ( $\mathrm{p}$-value 0,0002) con respecto al uso actual del suelo de la cuenca.

De acuerdo con la figura 7, para el escenario actual se reportó un caudal promedio de $14,86 \mathrm{~m}^{3} / \mathrm{s}$, entre un rango de 9,21 y $21,28 \mathrm{~m}^{3} / \mathrm{s}$, alcanzando su pico en el mes de marzo del año 2007. Para el escenario B1 (positivo), el modelo obtuvo un caudal promedio de $21,89 \mathrm{~m}^{3} / \mathrm{s}$, con un rango de 7,99 a $44,65 \mathrm{~m}^{3} / \mathrm{s}$, con un pico en el mes de septiembre del año 2005, y, finalmente, para el escenario A2 (negativo), el modelo calculó un caudal promedio de $28,17 \mathrm{~m} 3 / \mathrm{s}$, oscilando entre un rango de 9,54 y $52,87 \mathrm{~m}^{3} / \mathrm{s}$. 


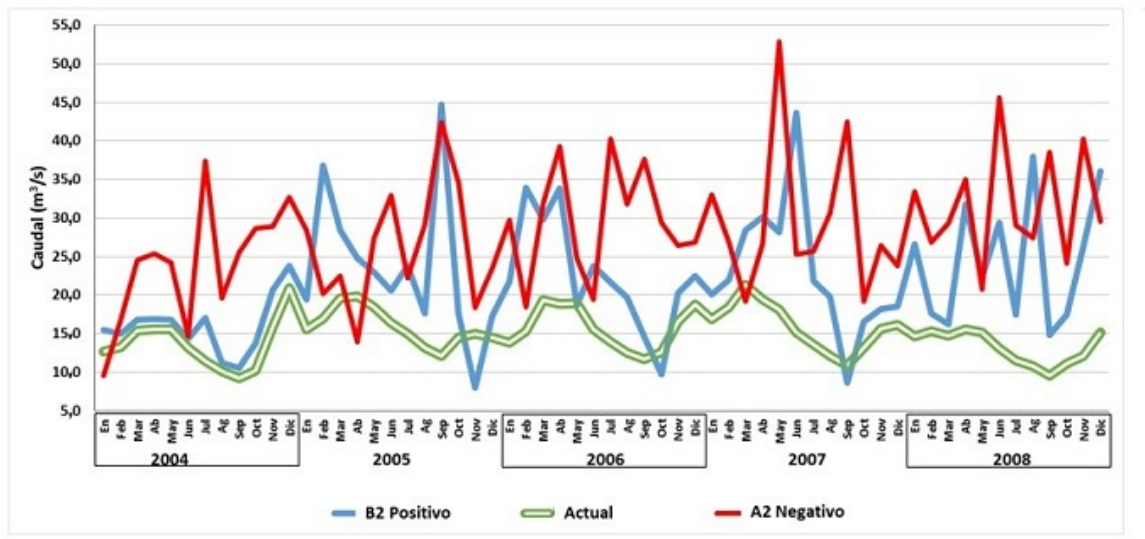

FIGURA 7.

Escurrimiento superficial de los diferentes escenarios de cambio climático en el distrito de drenaje del Valle de Sibundoy Fuente: elaboración propia

\section{Simulación de sedimentos con base en escenarios de cambio climático}

En los escenarios propuestos modelados se encontraron diferencias estadísticas significativas ( $\mathrm{p}$-value 0,0037). A continuación, se muestran los resultados de la producción de sedimentos, en $\mathrm{T} / \mathrm{ha} / \mathrm{a}$, de la cuenca frente a los escenarios generados a partir de modelos climáticos, lo que de esta manera permite compararlos con la situación actual.

Como puede verse en la figura 8 , para el escenario actual se reportó una producción de sedimentos promedio de $1,18 \mathrm{~T} / \mathrm{ha} / \mathrm{a}$. Para el escenario B1 (positivo), el modelo obtuvo una producción promedio de $3,13 \mathrm{~T} / \mathrm{ha} / \mathrm{a}$, con un rango entre 0,27 y $11,58 \mathrm{~T} / \mathrm{ha} / \mathrm{a}$, y para el escenario A2 (negativo), el modelo calculó una producción promedio de 5,78 T/ha/a, que oscila entre un intervalo de 0,53 y $14,57 \mathrm{~T} / \mathrm{ha} / \mathrm{a}$.

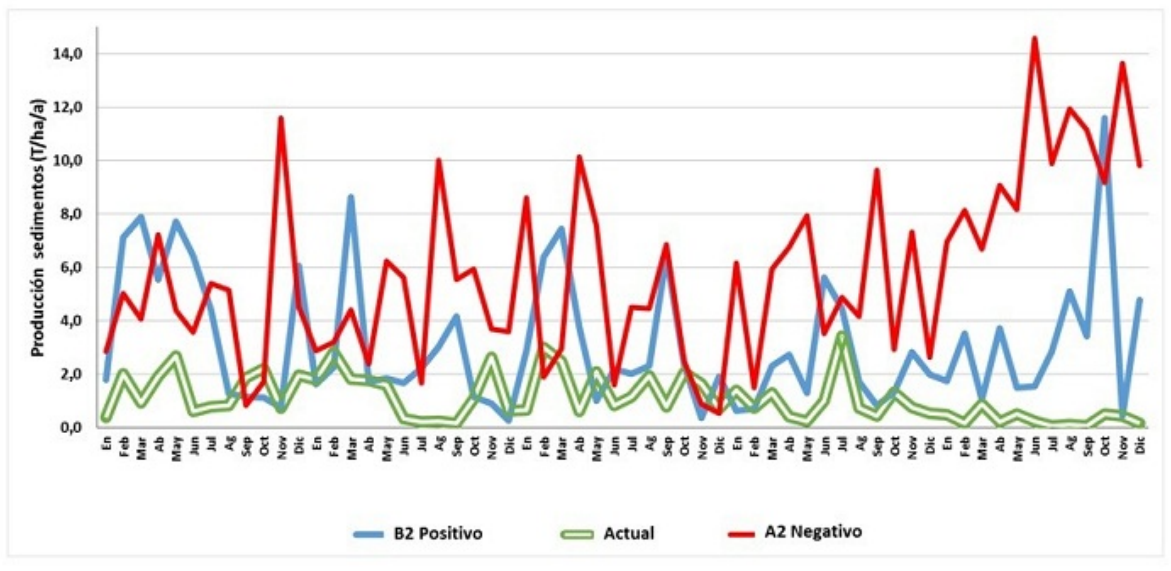

FIGURA 8.

Producción de sedimentos en los escenarios de cambio climático en el distrito de drenaje del Valle de Sibundoy Fuente: elaboración propia 


\section{Discusión}

\section{Balance hidrológico de la cuenca}

Al comparar los datos del balance hidrológico de este estudio con los reportados por Solarte (2014) en su investigación en la microcuenca Peñas Blancas, se encontró que el distrito de drenaje presenta una evapotranspiración del 21,84\%, mientras que en Peñas Blancas es del 51,24\%; a su vez la microcuenca reporta una salida de caudal en forma de escorrentía del 47,95\%, en contraste con el 75,03\% que se encontró para el distrito de drenaje. Estas diferencias marcadas se podrían explicar porque la zona de Peñas Blancas es un ecosistema paramuno, con alta capacidad para interceptar, almacenar y regular los flujos hídricos superficiales y subterráneos (Ministerio del Medio Ambiente, 2002), mientras que el distrito de drenaje del valle de Sibundoy, por su relieve y precipitación, se define como una zona de alta humedad y de climas fríos lluviosos, que tiene unas precipitaciones unimodales biestacionales para los meses de abril a agosto; por eso, su alto porcentaje de salida de agua en forma de escorrentía y su alta humedad, con pico de valores medios en los meses de junio-julio, los cuales pueden alcanzar hasta el 88,3\%, reiteran estas marcadas diferencias (Instituto de Hidrología, Meteorología y Estudios Ambientales [Ideam], 2006).

Por otra parte, los valores del balance hidrológico calculados con el modelo SWAT son menores a los hallados por Corpoamazonia (2008), que utilizó el método Thornthwaite-Mather. Esta entidad reportó valores promedio de precipitación de $1718,45 \mathrm{~mm}$ y un escurrimiento de $80,24 \mathrm{~m}^{3} / \mathrm{s}$. Además, si se analiza la serie de datos que Corpoamazonia utilizó para la precipitación del periodo 1970-1990, su promedio es mucho mayor a la serie de datos que se empleó en este estudio para el periodo 1993-2008; asimismo Corpoamazonia tuvo en cuenta más estaciones meteorológicas, repartidas en la parte plana, media y alta del distrito de drenaje, en contraste con las dos estaciones consideradas para este estudio.

\section{Calibración y validación del modelo para caudal}

Partiendo del hecho de que en el distrito de drenaje no se había realizado una simulación con los requerimientos del modelo SWAT, la importancia de este estudio radica en que, al conocer la relación entre lluvia y el caudal, se detalla la producción y disponibilidad del recurso agua y su interrelación con las coberturas y pendientes para la generación de sedimentos con base en la información climatológica histórica (Martínez, Rodríguez e Ibáñez, 2015).

En esta investigación el error de ajuste entre los caudales observados y simulados luego del proceso de calibración y validación fue del $17 \%$. La principal discrepancia entre los caudales observados y simulados, según Uribe, Quintero y Valencia (2013), se presenta en los ajustes de los caudales máximos, porque se realiza un solo registro de caudal por día, y es posible que no se alcancen a registrar las crecidas, que pueden presentarse durante las 24 horas del día. Solarte (2014) complementa esta hipótesis al afirmar que las diferencias entre los caudales observados y simulados se deben a que el modelo se diseñó para zonas templadas, mas no para el trópico, por lo que las condiciones biofísicas cambian significativamente, afectando de esta manera el comportamiento hidrológico de la microcuenca.

Esta investigación obtuvo un $\mathrm{R}^{2}$ de 0,83 y un NSE de 0,76 para la calibración y la validación de caudales, lo que cataloga a la simulación como muy buena, según la escala de valor propuesta por el modelo.

Al comparar el desempeño del modelo SWAT en algunas cuencas similares al distrito de drenaje del Valle de Sibundoy, se podría mencionar la cuenca del río Salado, en Buenos Aires (Argentina), que tiene un relieve con una pendiente suave, en la cual Brandizi y Labraga (2012) obtuvieron un $\mathrm{R}^{2}$ de 0,80 y un NSE de 0,50, lo que califica a esta simulación como satisfactoria. Cabe la pena anotar que estos mismos autores expresan 
que para cuencas con bajas pendientes la simulación puede ser más compleja, porque el drenaje natural está pobremente desarrollado. Es necesario recalcar que cuando en una cuenca se realizan cambios de coberturas de protección por ampliación de la frontera agropecuaria, según Rodríguez, Mojica, Caballero, Franco y Domínguez (2009), esto incide en una menor permeabilidad del suelo y un aumento en la escorrentía, lo que se comprobó experimentalmente en la cuenca del río David, en Panamá, donde se obtuvieron valores de $\mathrm{R}^{2}$ iniciales de 0,80 y un caudal de salida de $87,75 \mathrm{~m}^{3} / \mathrm{s}$; después de diez años, este valor disminuyó a un $\mathrm{R}^{2}$ de 0,67 y un caudal de salida de $145,23 \mathrm{~m}^{3} / \mathrm{s}$. Rodríguez et al. (2009) explican esta tendencia por el crecimiento urbanístico y por el aumento del área para usos pecuarios en la zona.

\section{Calibración y validación del modelo para sedimentos}

Teniendo en cuenta el cambio del uso del suelo que se determinó en el área de estudio, la generación de sedimentos se incrementó porque los cambios se dieron en zonas vulnerables, como terrenos en alta pendiente y praderizados. Conviene afirmar que Gutiérrez (2004) describe que las mayores producciones de sedimentos se dan cuando existen conflictos en el uso de suelo, lo que genera un aumento del movimiento del suelo por arrastre.

Los valores estadísticos para la simulación hidrológica de sedimentos que se reportan en esta investigación calcularon un $\mathrm{R}^{2}$ de 0,75 y un NSE de 0,68 , valores que ubican a la simulación dentro del rango de buena, de acuerdo con la categorización del modelo.

Partiendo del hecho de que en la zona de estudio uno de los limitantes fue la disponibilidad de los datos observados para la producción de sedimentos en la desembocadura de la cuenca, se tuvo realizar un proceso de depuración preliminar, lo que redujo la cantidad de datos disponibles, y en cierta medida eso explica por qué el $\mathrm{R}^{2}$ es menor con relación a la modelación de caudales. Además, Londoño y Zapata (2013) sostienen que la simulación de sedimentos es más compleja porque el modelo estima el potencial erosivo, el cual generalmente es mayor a la erosión real generada, y lo estima para toda la cuenca, suponiendo que el 100\% del sedimento generado llega a la desembocadura, aunque se sabe de antemano que esto no ocurre en la realidad, porque la cuenca actúa como un depósito que almacena sedimentos, sobre todo en las secciones donde la velocidad media es baja, lo que favorece la sedimentación de las partículas de mayor tamaño.

Otro aspecto relevante en el desarrollo de esta investigación fue establecer un valor numérico para la generación de sedimentos, porque hasta el momento los estudios en la zona se han basado en el análisis de los efectos, tal como lo menciona Corpoamazonia (2008), que concluye que el manejo del distrito de drenaje se enfoca en sacar sedimentos de los canales sin realizar acciones efectivas en las zonas de ladera en donde se genera una degradación progresiva por la ampliación de la frontera agropecuaria. En ese sentido, esta investigación a través del modelo SWAT estimó que la producción promedio de sedimentos para el distrito de drenaje estudiado fue de 1,18 T/ha/a. Al comparar el valor obtenido en este estudio con el de Gutiérrez (2004), de acuerdo con la clasificación de la degradación de los suelos, este se encuentra en la categoría baja, porque su valor es menor de $10 \mathrm{~T} / \mathrm{ha} / \mathrm{a}$; no obstante, hay que continuar con los planes de manejo ambiental dentro de la cuenca, porque este tipo de erosión aumenta rápido y es capaz de arrancar materiales del suelo por medio de la fuerza hidráulica de su flujo.

\section{Simulación de caudales con base en escenarios de cambio climático}

La simulación hidrológica permitió crear escenarios hipotéticos en los que se supone el comportamiento de la cuenca de acuerdo con nuevas coberturas y condiciones climatológicas. Esta predicción debe obedecer a consideraciones de las políticas agropecuarias, las tendencias de uso de la tierra y los conflictos en el uso 
del suelo, que permitirán tomar decisiones basadas en criterios técnicos (Cacoango, 2014). Los resultados obtenidos en función del escurrimiento superficial de la cuenca mostraron que el escenario positivo (B1) $\left(21,89 \mathrm{~m}^{3} / \mathrm{s}\right)$ y el negativo (A2) $\left(28,17 \mathrm{~m}^{3} / \mathrm{s}\right)$ presentaron un mayor escurrimiento que el obtenido con las coberturas actuales de la cuenca $\left(14,86 \mathrm{~m}^{3} / \mathrm{s}\right)$. En términos generales, esto se debe a que, al disminuir las coberturas de protección, el suelo pierde su capacidad de percolar y, por ende, su capacidad de almacenar agua, evacuando la mayor cantidad de agua en el momento de la precipitación, lo que puede generar problemas de evacuación e inundaciones (Escamilla, 2016). Esto también es soportado por Ataroff y Fermin (2000), quienes mencionan que un efecto a corto plazo de la remoción de bosques consiste en un aumento en el volumen anual de escurrimiento superficial. Este fenómeno ha sido reportado por la Organización de las Naciones Unidas para la Alimentación y la Agricultura (FAO) (2016), la cual manifestó que, cuando se produce la alteración de las condiciones edáficas y de coberturas, pueden producir un sello superficial en el suelo que disminuye sustancialmente su capacidad de infiltración, considerando a la escorrentía superficial como el principal agente de transporte de sedimentos. Cuando se estimó que para el año 2046 disminuirán las coberturas de bosque y se ampliarán las áreas destinadas para actividades agropecuarias y urbanísticas dentro de la cuenca, se estimó también que habrán mayores escorrentías superficiales, aspecto que puede traer impactos ambientales, como inundaciones, porque se rebasará la capacidad de diseño hidráulico de los canales, ya que, según las HRU calculadas por el modelo SWAT, cuando los valores medios superan $10535 \mathrm{~m}^{3} / \mathrm{s}$ la probabilidad de que se produzca una inundación es mayor.

Gentry y López (citados por Solarte, 2014) indican que, cuando en las cuencas de los ríos Ucayali y Marañón se han evidenciado procesos de deforestación, estos se han relacionado con el incremento de los caudales de los ríos en Iquitos y de sus ríos tributarios del Perú. Bruijnzeel (2004) reafirma esta hipótesis, porque menciona que cuando hay una conversión de bosque a otro uso de tierra, generalmente menos demandante de agua, esto hace que exista más agua disponible en el suelo y que se produzcan incrementos en el volumen anual de escorrentía. En este estudio, la línea evolutiva A2, considerada como negativa, obtuvo un mayor caudal que la B1, considerada como positiva, aspecto que se explica por la relación directa de las coberturas con el almacenamiento o escorrentía, lo que muestra cierta similitud con Cacoango (2014) en el modelamiento de la microcuenca del río Illangama, en el que el escurrimiento superficial aumentaba al sustituir áreas de bosque por potreros, pasando de 5,8 a 7,25 $\mathrm{m}^{3} / \mathrm{s}$. Asimismo, Crespo et al. (2008) determinaron la influencia de la relación entre caudales y coberturas: cuando la cuenca tenía una cobertura de $90 \%$ de bosque y $10 \%$ de pastos, se obtuvo un flujo de base de $3,04 \mathrm{~m}^{3} / \mathrm{s}$, y en el escenario donde se simuló un $100 \%$ de pastos se reportaron valores de $5,2 \mathrm{~m}^{3} / \mathrm{s}$ para escurrimientos superficiales.

\section{Simulación de sedimentos con base en escenarios de cambio climático}

Cuello (2003) encontró que las cuencas en condiciones de sobreuso producen la mayor cantidad de sedimentos y que las cuencas donde se encuentra la mayor cantidad de bosques son menos propensas a la erosión.

Para comenzar el análisis de los datos que se obtuvieron en este estudio, es conveniente reiterar la marcada tendencia de la producción de sedimentos cuando se aumentan las áreas de la frontera agropecuaria y urbanística. Específicamente, aquí se reporta para las condiciones actuales una producción media de 1,18 $\mathrm{T} / \mathrm{ha} / \mathrm{a}$; para la línea positiva, 3,13 T/ha/a, y para la línea negativa, 5,78 T/ha/a. Estos resultados siguen la tendencia de los reportados para la simulación de caudales de Brea y Balocchi (2010): la generación de sedimentos se encuentra directamente relacionada con el caudal, porque la velocidad mínima para desprender y transportar una partícula de suelo está en función del diámetro de esta; sin embargo, una vez que la partícula ha sido desprendida, se necesitará menos energía para que siga en movimiento dentro del cauce. 
Estos resultados van de la mano con los de Ríos (2015), quien estimó el balance hídrico y la producción de sedimentos en la subcuenca ganadera del río Jabonal, y encontró una relación similar a la de este estudio, en donde, al disminuir las coberturas de protección, observó cómo se incrementó la producción de sedimentos. Este mismo autor demostró que se puede disminuir hasta en un $42 \%$ la pérdida de suelo por erosión a través de la implementación de sistemas silvopastoriles dentro de un plan sostenible de manejo de esta cuenca.

Igualmente, Roco (2014) encontró una situación similar en la cuenca de Los Ulmos, donde observó que en el período de poscosecha, en el "suelo desnudo" fue mayor la carga de sedimento fino simulado por SWAT por arrastre y la producción de sedimentos provenientes de laderas forestales a la de un escenario con cobertura vegetal (sin intervención).

En contraposición a los datos reportados en esta investigación, Osuna-Osuna, White, Díaz-Torres y DávilaVásquez (2015), en un estudio en el río Tecolutla simulando escenarios de conservación del suelo, mostraron que cuando se realiza una rotación de cultivos se puede obtener una reducción en la producción de sedimentos de hasta el 1,08\%. Esto reitera, una vez más, la marcada relación entre generación de sedimentos y coberturas de protección simuladas a través de escenarios de cambio climático.

\section{Conclusiones}

Con la implementación del modelo SWAT para la modelación hidrológica del distrito de drenaje del Valle de Sibundoy, se determinó que la generación de sedimentos en la cuenca es de 1,18 T/ha/a, con la calibración y validación del modelo para las asociaciones de suelo, coberturas actualizadas y variables climatológicas para un periodo de retorno de quince años.

Con las variables climáticas del modelo GFDL-CM2.0, se determinó que, en condiciones de sobreuso en las coberturas, la cuenca podría alcanzar valores de 3,13 T/ha/a para el escenario B1 (bajo), y para el escenario A2 (alto) de 5,78 T/ha/a, lo que produciría una notable disminución de la capacidad hidráulica de los canales y generaría inundaciones.

La simulación hidrológica calculó que el caudal promedio mensual para la cuenca del distrito de drenaje del Valle de Sibundoy en la garganta del Balsayaco es de $32,26 \mathrm{~m}^{3} / \mathrm{s}$, distribuidos entre los aportes del canal $\mathrm{B}$, con $16,89 \mathrm{~m}^{3} / \mathrm{s}$; el canal A, con $11,96 \mathrm{~m}^{3} / \mathrm{s}$, y el cauce viejo del río Putumayo, que entrega $3,41 \mathrm{~m}^{3} / \mathrm{s}$, con un $\mathrm{R}^{2}$ de 0,83 y un NSE de 0,76 .

$\mathrm{Si}$ en el distrito de drenaje continúan con las prácticas de manejo actuales, en diecinueve años la producción de sedimentos aumentará alrededor del $200 \%$, lo que acarreará inminentes problemas ambientales, como degradación de la capa vegetal y erosión severa, de manera que se pondría en riesgo la vida y las propiedades de los habitantes de esta cuenca.

La construcción del distrito de drenaje condujo a la modificación de todo el régimen hidrográfico del río Putumayo con el fin de habilitar una zona naturalmente inundable para actividades agropecuarias. Este aspecto enmarca la vulnerabilidad cuando ocurren altas precipitaciones, que, sumado al manejo inadecuado de zonas de laderas, ha ocasionado la colmatación de los canales y creado un círculo entre causa y efecto que hace más álgida esta problemática dentro del distrito. En ese orden de ideas, la generación de alternativas de manejo sostenible en el marco de la conservación de suelos, sistemas productivos sustentables, restauración de coberturas y gestión del riesgo en el recurso hídrico tendientes a reducir la generación de sedimentos requiere la participación activa de los actores directos del distrito de drenaje, la institucionalidad y la autoridad ambiental para garantizar el dinamismo y la efectividad de estas. 


\section{Referencias}

Ataroff, M., y Fermin, R. (2000). Deforestation Impact on Water Dynamics in a Venezuelan Andean Cloud Forest. Venezuela: Ambio.

Brandizi, L., y Labraga, J. (2012). Calibración del modelo hidrológico SWAT en la cuenca del río Salado, provincia de Buenos Aires. Buenos Aires: Centro Nacional Patagónico (Cenpat).

Brea, J., y Balocchi, F. (2010). Procesos de erosión-sedimentación en cauces y cuencas. Montevideo: Unesco. Recuperado de https://www.unesdoc.unesco.org/images/0021/002163/216338s.pdf

Bruijnzeel, L. (2004). Hydrological functions of tropical forests: Not seeing the soil for the trees. Agricultura, Ecosystems \& Environment, 104(1), 185-228. https://doi.org/10.1016/j.agee.2004.01.015

Cacoango, J. (2014). Modelamiento de la microcuenca del río Illangama con la utilización del modelo hidrológico soil and water assessment tool "SWAT". Quito: Universidad Central del Ecuador.

Corporación para el Desarrollo del Sur de la Amazonia (Corpoamazonia). (2008). Plan de Ordenación y manejo de la cuenca alta-alta del Rio Putumayo. Mocoa: Corpoamazonia.

Corporación para el Desarrollo del Sur de la Amazonia (Corpoamazonia) y Fundación Cultural del Putumayo. (2007). Plan de manejo ambiental de los humedales de la parte plana del valle de Sibundoy. Mocoa: Corpoamazonia.

Cortez, A. (2004). Suelos colombianos, una mirada desde la academia. Bogotá: Fundación Universidad Jorge Tadeo Lozano.

Crespo, P., Coello, C., Iñiguez, V., Cisneros, F., Cisneros, P., Ramírez, M., y Feyen, J. (2008). Evaluación de SWAT 2000 como herramienta para el análisis de escenarios de cambio de uso del suelo en microcuencas de montaña del sur del Ecuador. Ponencia presentada en el XI Congreso Ecuatoriano de la Ciencia del Suelo, Quito, Ecuador, 29-31 de octubre. Recuperado de https://www.secsuelo.org/wp-content/uploads/2015/06/2.-Ing.-Patricio-Cr espo.-Swat2000.pdf

Cuello, M. (2003). Estimación de la producción y transporte de sedimentos en la cuenca alta del río Yaque del norte y del río Guanajuma, República Dominicana. Turrialba: Centro Agronómico Tropical de Investigación y Enseñanza (Catie).

Escamilla, V. (2016). Manual operativo de modelo SWAT. México: Colegio de la Frontera Sur (Ecosur).

Gutiérrez, M. (2004). Simulación de la escorrentía y producción de sedimentos aplicando SWAT en las microcuencas 7 y 13, subcuenca III, cuenca sur del lago Managua, Nicaragua. Managua: Universidad Nacional Agraria.

Instituto de Hidrología, Meteorología y Estudios Ambientales (Ideam). (2006). Zonificación ambiental en el ordenamiento de cuencas hidrográficas en Colombia. Bogotá: Ideam.

Londoño, F., y Zapata, S. (2013). Variabilidad espacial de los sedimentos en la cuenca del embalse La Fe. Envigado: Escuela de Ingeniería de Antioquia.

Martínez, L., Rodríguez, I., e Ibáñez, L. (2015). Calibración del modelo SWAT, en la cuenca del río Turbio, Guanajuato. Ponencia presentada en el I Congreso Iberoamericano sobre Sedimentos y Ecología, Querétaro, México, julio 21-24. Recuperado de https://www.dokumen.tips/documents/calibracin-del-modelo-swat-en-la-cuenca-atlorg mxisi-lacimages1er-congresoarticuloscalibracion-della.html

Ministerio del Medio Ambiente. (2002). Programa para el desarrollo sostenible y restauración de ecosistemas de alta montaña colombiana. Bogotá: Imprenta Nacional de Colombia. Recuperado de https://www.minambiente.gov.co/images/BosquesBiodiversidadyServiciosEcosistemicos/pdf/Paramos/55 95_250510_rest_alta_montana_paramo.pdf

Neitsch, S., Arnold, J., Kiniry, J., y Williams, J. (2005). Herramienta de evaluación de suelo y agua. Documentación teórica. Temple: Agricultural Research Service.

Organización de las Naciones Unidas para la Alimentación y la Agricultura (FAO). (2016). Erosión y pérdida de fertilidad del suelo. Recuperado de https://www.fao.org/docrep/t2351s/T2351S06.htm

Osuna-Osuna, A., White, D., Díaz-Torres, J., y Dávila-Vásquez, G. (2015). Estimación de la carga de sedimentos en la cuenca del río Tecolutla utilizando el modelo SWAT. Ponencia presentada en el I Congreso Iberoamericano 
sobre Sedimentos y Ecología, Querétaro, México, julio 21-24. Recuperado de https://www.docplayer.es/73270 210-Estimacion-de-la-carga-de-sedimentos-en-la-cuenca-del-rio-tecolutla-utilizando-el-modelo-swat.html

Ríos, J. (2015). Estimación del balance hídrico y producción de sedimentos bajo tres escenarios de coberturas en la subcuenca ganadera del río Jabonal, Costa Rica, mediante el empleo de SWAT. Turrialba: Cathie.

Roco, E. (2014). Uso del modelo SWAT para estudiar los efectos de cambios de uso del suelo en la hidrología, transporte de sedimento fino y en las fuentes de aporte de sedimentos en cuencas experimentales del sur de Chile. Valdivia: Universidad Austral de Chile.

Rodríguez, X., Mojica, V., Caballero, M., Franco, N., y Domínguez, V. (2009). Modelación hidrológica con escenarios de contaminación difusa, para la planificación ambiental en la cuenca del río David. Campus Victor Levi Sasso Panamá: Universidad Tecnológica de Panamá.

Secretaría Distrital de Integración Social. (2013). Guía metodológica para la evaluación de aspectos e impactos ambientales. Bogotá: Alcaldía Mayor de Bogotá D. C.

Solarte, J. G. (2014). Efecto del cambio de uso del suelo sobre la cantidad superficial del recurso hídrico en la microcuenca Peñas Blancas, municipio de Tangua, departamento de Nariño (tesis de maestría). San Juan de Pasto, Colombia: Universidad Mariana.

Srinivasan, R. (2013). Manual introductorio. Soil and water assessment tool. Texas: UPCT. Recuperado de de https: //www.upct.es/ minaeees/hidrologia.pdf

Uribe, N. (2010). Conceptos básicos y guía rápida para el usuario-versión SWAT 2005. Palmira: Centro Internacional de Agricultura Tropical (CIAT).

Uribe, N., Quintero, M., y Valencia, J. (2013). Aplicación del modelo hidrológico SWAT (Soil and Water Assessment Tool) a la cuenca del río Cañete. Cali: Centro Internacional de Agricultura Tropical (CIAT).

\section{Notas}

* Artículo de investigación.

Este artículo es el resultado del proyecto de investigación "Evaluación de la generación de sedimentos en el distrito de drenaje en el Valle de Sibundoy, departamento del Putumayo (Colombia)", desarrollado por el autor en los períodos 2016, 2017 y 2018 como trabajo de grado para optar al título de magíster en Ingeniería Ambiental de la Universidad Mariana de Pasto (Nariño).

\section{Licencia Creative Commons CC BY 4.0}

Cómo citar este artículo: Delgado-Gómez, M. D. (2019). Evaluación de la generación de sedimentos en el distrito de drenaje del Valle de Sibundoy, departamento del Putumayo (Colombia). Ambiente y Desarrollo, 23(44). https://doi.org/10.11144/Javeriana.ayd23-44.egsd 